\title{
Sexual Problems and the Gynaecologist: Are We Doing Enough?
}

\author{
1Pratibha Singh, ${ }^{2}$ Neha Mathur
}

\section{ABSTRACT}

Introduction: Sexual health is an important part of womens' life and wellbeing. Female sexual dysfunction is a complex problem involving an interplay of various physical, emotional, psychological factors often less understood by the general doctors. Gynecologists are many a time first contact person for the woman, where she comes for help. As there are very few or maybe no specialist in this field, in most of the developing countries, responsibility to adequately address and manage these issues in women comes to gynecologists. How does a gynecologist see these issues? Are they comfortable and confident in handling these problems? This study was undertaken to address all these issues.

Materials and methods: This was a questionnaire based study conducted on gynecologists in Jodhpur, Rajasthan and neighboring areas of Rajasthan, India. Gynecologists were contacted by personal contact or mail and were asked to fill out a questionnaire, which was analysed.

Results: Most gynecologists were females (95\%). The average duration of practice was 7.8 years. Most of the gynecologist $(77.8 \%)$ responded that they do not take a sexual history routinely. Gynecologists who were doing mainly infertility practice were more likely ( $80 \%$ vs $4.46 \%$ ) to take a sexual history. A total of $85.4 \%$ admitted that they do not feel confident in managing these problems, though they were open to discussion. They suggested that training during the graduation and postgraduation in this area could be helpful.

Conclusion: All gynecologists should include screening questions regarding sexual well-being as a standard of practice. The gynecologists should be comfortable in discussing the issue of sexual health with their patients and skilled to treat them. The academic medical organization should ensure that students have sufficient knowledge, attitude, and skills to address issues of sexual health.

Keywords: Gynecologist, Questionnaire based study, Sex education, Sexual problems

How to cite this article: Singh P, Mathur N. Sexual Problems and the Gynaecologist: Are We Doing Enough? J South Asian Feder Obst Gynae 2018;10(3):191-193.

\footnotetext{
${ }^{1}$ Professor, ${ }^{2}$ Consultant

${ }^{1}$ Department of Obstetrics and Gynecology, AlIMS, Jodhpur, Rajasthan, India

${ }^{2}$ Department of Obstetrics and Gynecology, Private Practice, Jodhpur, Rajasthan, India

Corresponding Author: Pratibha Singh, Professor, Department of Obstetrics and Gynecology, AlIMS Jodhpur, Jodhpur, Rajasthan, India, e-mail: drpratibha69@hotmail.com
}

Source of support: Nil

Conflict of interest: None

Date of received: 09/12/2017

Date of acceptance: 03/05/2018

Date of publication: December 2018

\section{INTRODUCTION}

Obstetricians and gynecologists are the womens' favored healthcare personnels' when talking about sexuality and so have to deal with women's sexuality every day in their offices. Reproductive health also encompasses that women have safe and satisfying sexual life, and are capable of reproduction as per their wishes. As gynaecologist we have to improve the quality of women's reproductive and sexual health.

Women's expectations of their doctors have changed over the past years. Many women associate sexual problems with physical problems and expect their gynecologist will be knowledgeable about these problems and comfortable in discussing it. ${ }^{1,2}$ Studies have shown that the many women feel that discussing sex with their doctor is fine and want information from the healthcare provider.

Doctors own attitude, discomfort, and reluctance to discuss these sexuality related issues; which may stem from their inadequate knowledge in the field of sexuality, and probably the concern of false allegation of inappropriate behavior in case of a male gynaecologist. Many societies consider sex as a taboo, and even married women do not talk about sexual matters. Gynecologists are also part of that same society, so talking about sex may be difficult for both doctor and women. Gynaecologist need to have their comfort, as well as confidence in discussing and treating sexual health issues.

Sexual dysfunctions were regarded in the past as manifestations of disordered psychology. Many studies done over past years, now suggests that sexual problems are not merely manifestations of profound emotional disturbance, they commonly occur in persons who are otherwise well and have no other psychological symptoms. But yet there is not enough concern over this problem in gynecologists to consider it as a legitimate health problem which deserves education, service or research. 
Sexual problems can originate from multiple organ systems as the pelvis is a limited space occupied by many organs-bladder, rectum, uterus, ovaries, etc. Deep dyspareunia can be caused by endometriosis, interstitial cystitis, adnexal pathology, retroverted uterus, shortened vagina, pelvic floor hypertonicity, over vigorous penetration, postsurgical scarring of a vault or intraperitoneal scarring, prolapse, excessive penile length, pelvic congestion syndrome, constipation, or other bowel issues. It remains unclear whether uterine myomas and adenomyosis are causes of deep dyspareunia. ${ }^{3,4}$ Besides these many systemic diseases and their treatment may cause sexual problems. The prevalence of sexual concerns, spanning desire, arousal, and orgasmic dysfunction is two to three times greater in women with diabetes than in women in control groups (75\% vs 30.6\%). ${ }^{5}$ Women with hypertension may be twice as likely as normotensive women to experience sexual dysfunction ( $42.1 \%$ vs $19.4 \%$ ), which can be reduced by effective treatment of their blood pressure. $^{6}$

The biological event, e.g., childbirth, postpartum, menopause, etc., many also affect her sexuality. ${ }^{7}$ Women may have problems with sexual desire, sexual arousal, and/or orgasmic experience; they also may experience sexual pain disorders, including dyspareunia and vaginismus. According to the biopsychosocial model, biological, psychological, interpersonal, and cultural factors may all play a role in the development and perpetuation of sexual disorders. ${ }^{2,8}$

Limbic system with its connection to hypothalamus, thalamus, anterior and medial frontal cortex is core to normal sexual function. Sexual desire is initiated in the brain, which has been primed with sex steroids, including androgens, estrogens, and progestins. ${ }^{9,10}$ So a knowledge of different hormonal functions, medical, social and relationship problems, addressing to socio-cultural issues may greatly help in managing these complex issues. The medical skills a gynecologist uses in managing general health and illness play an important role in managing sexual concerns. Knowing how medical and surgical diseases and their treatment affect sexual function is essential.

This study was undertaken to know the attitude, the comfort of a general obstetrician and gynaecologist to address these problems and measures to improve it.

\section{MATERIALS AND METHODS}

This study was conducted on gynecologists of Jodhpur city and neighboring areas who were willing to participate. Gynecologists were contacted personally and by mail. They were given a simple questionnaire to fill; identity was not revealed. Willingness to fill the forms were taken as consent. These were later analyzed.

\section{RESULTS}

One hundred and thirty-seven gynecologists responded, were included in the study. Most (95\%) gynecologists were females $(130 / 137)$ as is common in this part of the country. The average duration of practice was 7.8 years. And the mean age was 40.1 years. Patients coming with sexual problems comprise $1.2 \%$ of the total patients seen by them.

A total of $77.8 \%$ of gynecologist responded that they do not take a sexual history routinely and only $18.2 \%$ (25/137) were enquiring about sexual concerns. Gynecologists who were doing mainly infertility practice were more likely to ask about sexual concerns-80\% (20/25) vs $4.46 \%$ (5/112). Very few patients came with complaints pertaining to sexual dysfunction $(1.2 \%)$ in their general practice. The common problems were dyspareunia, low desire, and less sexual contact. But if the patient had sexual concerns they $61.3 \%(84 / 137)$ were comfortable talking and discussing it; this was again more in those who were mainly doing infertility practice $96 \%(24 / 25)$ vs $53.6 \%(60 / 112)$.

Only $14.6 \%$ (20/137) of the gynecologist felt they were confident to manage sexual problems, most of them were those doing infertility practice. Majority of gynecologist $85.4 \%(115 / 137)$ admitted that they do not feel confident in handling these problems due to multiple reasons, most importantly due to lack of training and exposure and referred them to psychiatrist/psychologist and sometimes to physicians.

They suggested that training during the graduation and postgraduation in this area could be helpful.

\section{DISCUSSION}

A study published in 2010 by Abdolrasulnia et al. ${ }^{11}$ on primary care physician and gynecologist, 21\% of OB/ GYN and $38 \%$ of primary care physicians stated they were not at all confident in treating sexual dysfunction. This incidence was found to be lower in our study.

Kottmal et al. in 2014 published their study where $7.9 \%$ of the respondents routinely explored sexual issues with more than $80 \%$ of their patients, ${ }^{12}$ as contrast to our study where $18.2 \%$ enquire about sexual issues, mostly doing infertility practice as it is an important issue in those group of patients. This may also be due to differences in socio-cultural norms in these two different geographic areas. Even Abdolrasulnia et al. ${ }^{11}$ found that $40.4 \%$ reported having at least brief (at least 1-2 days) of training in sexual medicine, while none of the gynecologists in our study had any training in these areas, which might also have contributed to lower percentage exploring the sexual health concerns.

Briedite et al., in their study, found that only one-third of the patients had ever been asked about their sexual 
life by a gynecologist while the majority $(80 \%)$ of the respondents reported they would like to be asked about and discuss sexual issues. ${ }^{13}$ The patients mostly did not complain because of psycho-emotional barriers, and shame was the main barrier for patients to talk about their problems. In the present study, this factor was not assessed.

While many factors were identified for this lack, a few among them being time constraints, the perceived lack of effective therapies, perceptions regarding patientphysician gender discordance. ${ }^{11}$ In our study major cause was gynecologist do not feel confident in managing these problems as they have insufficient exposure and training. This could be very well taken care of by including these areas in the medical curriculum in the postgraduate training programme. Most of the problems can be effectively addressed by simple measures and medication, counseling; only a few of them require referrals. At a minimum, the clinician needs to assess the domains of sexual desire, arousal, orgasm, and pain from the women and partner if needed. ${ }^{1,2}$

In our study, gynecologists are comfortable in discussing sexual problems but not that confident in managing them, and this tells us the importance of training and exposure in their medical school to address these issues. CMEs and short-term training might also be helpful in rectifying this.

\section{CONCLUSION}

Female sexual dysfunctions are of concern to women and manifest in a variety of ways. Clinicians may fail to screen, diagnose, or treat them, and may not make appropriate referrals. The assessment of sexual health is insufficient in gynecological care, and sexual history-taking and evaluation of sexual functions should be included in routine gynecological health assessments. ${ }^{1,13}$ While gynecologists are comfortable discussing sexual concerns with their patients; but not that confident in treating them due to lack of exposure and knowledge in this field. Academic organizations should ensure that students get adequate knowledge and exposure in managing common sexual problems.

\section{REFERENCES}

1. Yulevitch A, Czamanski-Cohen J, Segal D, Ben-Zion I, Kushnir T. The vagina dialogues: genital self-image and communication with physicians about sexual dysfunction and dissatisfaction among Jewish patients in a women's health clinic in southern Israel. J Sex Med. 2013 Dec;10(12):30593068.

2. Lamont J, Bajzak K, Bouchard C, Burnett M, Byers S, Cohen $\mathrm{T}$, et al. Female Sexual Health Consensus Clinical Guidelines. Journal of Obstetrics and Gynaecology Canada [Internet]. Elsevier BV; 2012 Aug;34(8):S1-S4.

3. Ferrero S, Abbamonte LH, Giordano M, Parisi M, Ragni N, Remorgida V. Uterine myomas, dyspareunia and sexual function. Fertil Steril 2006;86(5):1504-1510.

4. Ferrero S, Ragni N, Remorgida V. Deep dyspareunia: causes, treatments, and results. Curr Opin Obstet Gynecol 2008; 20(4):394-399.

5. Palacios S, Castano R, Grazziotin A. Epidemiology of female sexual dysfunction. Maturitas 2009;63:119-123.

6. Doumas M, Tsiodras S, Tsakitis A, Douma S, Chounta A, Papadopoulos A, et al. Female sexual dysfunction in essential hypertension: a common problem being uncovered. J Hypertens 2006;24:2387-2392

7. Khajehei M. Sexuality after childbirth: Gaps and needs. World J Obstet Gynecol 2012;1(2):14-16.

8. Althof SE, Leiblum SR, Chevret-Measson M, Hartmann U, Levine SB, McCabe M, et al. Psychological and interpersonal dimensions of sexual function and dysfunction. J Sex Med 2005;2:793-800

9. Pfaus JG. Pathways of sexual desire. J Sex Med 2009; Jun;6(6): 1506-1533.

10. Rees PM, Fowler CJ, Maas CP. Sexual dysfunction 2: sexual function in men and women with neurological disorders. Lancet 2007;369:512-525

11. Abdolrasulnia M, Shewchuk RM, Roepke N, Granstaff US, Dean J, Foster JA, Goldstein AT, Casebeer L. Management of female sexual problems: perceived barriers, practice patterns, and confidence among primary care physicians and gynecologists J Sex Med. 2010 Jul; 7(7):2499-2508

12. Kottmel A, Ruether-Wolf KV, Bitzer J. Do gynecologists talk about sexual dysfunction with their patients? J Sex Med. 2014 Aug 11(8):2048-2054.

13. Briedite I, Ancane G, Ancans A, Erts R. Insufficient assessment of sexual dysfunction: a problem in gynecological practice. Medicina (Kaunas). 2013;49(7):315-320. 